\title{
Die Puure-Huus Gruppe: Speerspitze der Basis gegen den eigenen Frust
}

Andreas Girr

Infogruppe für Freipraktizierende

Korrespondenz:

Dr. med. Andreas Girr Waldstrasse 18 CH-8132 Egg

info[at]puure-huus.ch www.puure-huus.ch

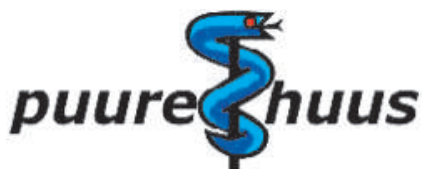

Ist Ihnen das Gefühl der Ohnmacht und der Resignation vertraut? Machen Sie auch immer wieder die Faust im Sack, ohne Ihrem Ärger Luft zu verschaffen? Haben Sie auch die Nase voll, in den Medien ständig als Buhmann der Nation missbraucht zu werden? Arbeiten Sie gerne zum Tarifansatz wie vor 30 Jahren? Lieben Sie das Wort «kostenneutral»?

\section{Wir helfen Ihrer Faust aus dem Sack}

Wir von der Puure-Huus Gruppe haben uns das Ziel gesetzt, weder an einem Magenulcus noch an einem Burn-out zu erkranken, sondern die Dinge, die uns beschäftigen, ohne Rücksicht auf die politische Raison beim Namen zu nennen. In diesem Zusammenhang haben wir auch schon das Vergnügen gehabt, ungefragt an prominenter Stelle in der Zeitung zu erscheinen. Wir vertreten die Anliegen der ganzen Ärzteschaft, jedoch liegt aufgrund der aktuellen Zusammensetzung unserer Gruppe der Schwerpunkt bei den frei praktizierenden Ärzten.

Das Puure-Huus: Bereit für jeden «Hoselupf» Die Puure-Huus Gruppe ist 2001 in Wermatswil, im Restaurant Puurehuus gegründet worden, von dort stammt unser Name. Damals versuchten die Apotheker die direkte Medikamentenabgabe (DMA) im Kanton Zürich abzuwürgen. Wir haben deshalb, ganz am Anfang der Internetnutzung, mit Mails unsere Kollegen zum Widerstand und zur Mitarbeit aufgerufen. Es ist uns gelungen, ein «feu sacré» zu entfachen, das uns bei den Abstimmungen 2001, 2003 und 2008 dreimal zum Sieg verholfen hat. Nach der Einführung der DMA im ganzen Kanton Zürich am 1. Mai 2012 stellte sich für uns die Frage, ob wir uns nach erreichtem Ziel auflösen oder andere Problemfelder bearbeiten wollen.

\section{Wir müssen unser eigenes Gärtchen engagiert pflegen - Niemand tut es für uns}

Wir haben uns entschlossen, in Anbetracht der politischen Lage weiter zu kämpfen. Neben den kleinen Tagesärgernissen, zu denen wir gerne Infos und Tipps geben, haben wir uns folgende politischen Ziele von gesamtschweizerischem Interesse auf die Fahne geschrieben:
- Kampf gegen die Einheitskasse

- Wiedereinführung des ambulanten Privattarifs

- Erhalt und Ausbau der DMA (Kantone Aargau, Bern, Graubünden)

- Erhalt der Margen auf den Medikamenten

Das aggressive und undurchsichtige Gebaren vieler Krankenkassen hat die Prämienzahler und zahlreiche Ärzte in Rage gebracht. Ob der Rundumschlag «Einheitskasse» allerdings die Lösung aller Probleme darstellt, wagen wir sehr zu bezweifeln. Schon jetzt ist der einzelne Arzt gegenüber den Krankenkassen in einer schlechten Position. Was aber erst, wenn es nur noch die eine Kasse gibt? Jeder von uns hat schon von Wirtschaftlichkeitsverfahren von santésuisse gehört, die für den betroffenen Arzt existentiell werden können. Wir dürfen nicht kampflos zulassen, dass diese Davidund-Goliath-Situation noch weiter verschärft wird!

Wenn die Krankenkassen selbst aus nachvollziehbaren Gründen gegen eine Einheitskasse sind, so heisst das nicht, dass wir aus eigenen Gründen nicht auch dagegen sein können. Überdies verfehlt die Initiative ihr eigentliches Ziel: Niemand, der sich mit der Materie eingehend befasst hat, erwartet eine Kostendämpfung.

Die Wiedereinführung des ambulanten Privattarifs, der mit der Einführung des KVG 1995 abgeschafft wurde, wäre eine Massnahme, die der frei praktizierenden Ärzteschaft einen Zusatzverdienst verschaffen würde, ohne die soziale Krankenkasse zu belasten. Weshalb der ambulante, nicht aber der stationäre Privattarif zu einer Zweiklassenmedizin führen soll, ist für uns nicht nachvollziehbar.

Die direkte Medikamentenabgabe (DMA) ist eine bei den Patienten sehr beliebte Form des Medikamentenbezugs und gehört zum Service public wie der Notfalldienst. Nach den Kantonen Zürich und Schaffhausen steht im Herbst 2013 im Kanton Aargau eine Abstimmung zur DMA an. Der mündige Bürger soll selber entscheiden, wo er die Medikamente bezieht - beim Arzt oder in der Apotheke. Wie ist es möglich, dass in elf Kantonen den Patienten diese Wahlfreiheit immer noch vorenthalten wird?

Der politische Druck auf die Medikamentenmargen besteht seit Jahren und wird weiter bestehen. Vergessen wir aber nicht, dass die Margen schon alleine durch das Sinken der Medikamentenpreise (z. B. durch Generikasubstitution) kleiner geworden sind. Haben Sie zudem einmal etwas von sinkenden Löhnen beim Personal gehört? Es ist stossend, wenn die Marge nur 


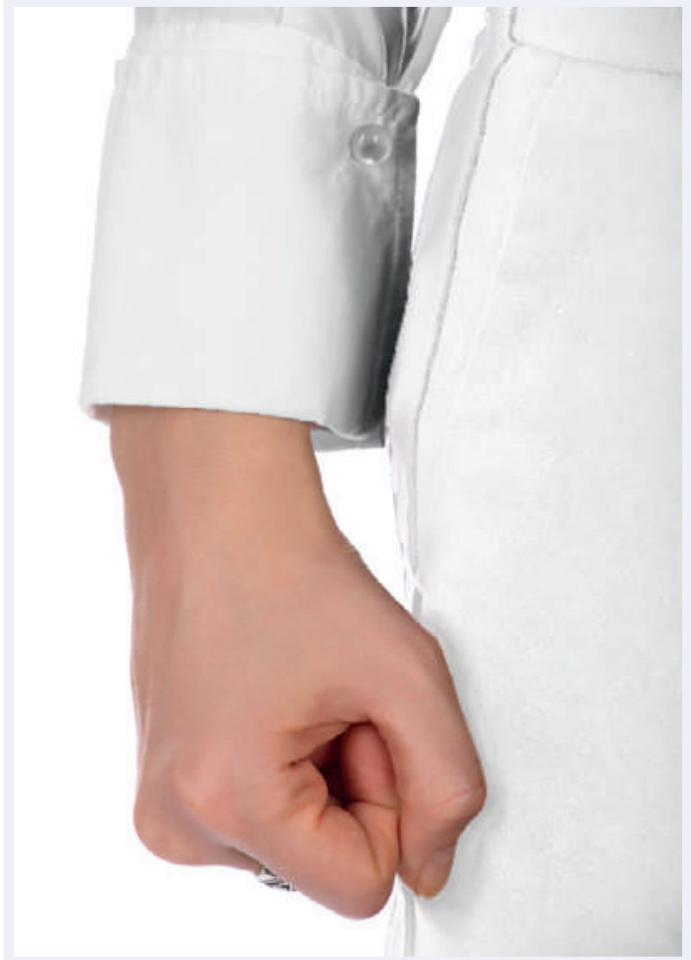

Das Motto von Puure-Huus: Nicht nur heimlich die Faust ballen, sondern Missstände aktiv bekämpfen. beim Arzt gesenkt werden soll, während die Apotheker neben den LOA-Zuschlägen auch noch eine höhere Marge erhalten sollen. Klar ist auf jeden Fall bereits jetzt: Die Medikamente sind beim Arzt billiger.

\section{Wer zahlt, befiehlt! Zahlt, wer befiehlt?}

Zum ersten Mal führt das Puure-Huus am 22. Oktober 2013 um 18.15 Uhr im Restaurant Doktorhaus in Wallisellen eine Podiumsdiskussion zu den Themen «Einheitskasse» und «ambulanter Privattarif» durch. Teilnehmen werden hochrangige Vertreter aus Politik, Krankenkassen und Standespolitik. Gesprochen wird vor allem übers Geld. Wir sind gespannt, ob die, die befehlen, auch wirklich bezahlen, oder ob in der Baugrube «Gesundheitswesen» die Ärztinnen und Ärzte weiterhin als «kostenneutrales Baumaterial» Verwendung finden. Nehmen Sie an diesem speziellen Anlass teil und diskutieren Sie mit!

\section{Bauer sucht Bäuerin}

Unsere Gruppe ist ein guteingespieltes Team, das seit Jahren erfolgreich zusammenarbeitet. Gerne würden wir in unserem Kreis engagierte Kolleginnen und Kollegen aus Praxis und Klinik, aus verschiedenen Ärztegenerationen und Fachrichtungen willkommen heissen. Wer krempelt die Ärmel hoch und arbeitet mit?

\section{Wer zahlt, befiehlt! Zahlt, wer befiehlt?}

Podiumsdiskussion am 22. Oktober 2013, 18.15 Uhr bis 21.00 Uhr, Wirtschaft zum Doktorhaus, Wallisellen Die Themen:

- Der ambulante Privattarif: Wege hinaus aus der Baugrube der Staatstarife

- Die Einheitskasse: Freiheit lebe wohl - Der Arzt als Funktionär

Referenten und Diskussionspartner:

- Nationalrätin Jacqueline Fehr, SP, Mitglied der Kommission Soziale Sicherheit und Gesundheit SGK

- Prof. Dr. med. Thomas Szucs, Präsident des Verwaltungsrates der Helsana

- Dr. sc. nat. Michael Willer, Mitglied der Konzernleitung Helsana

- Dr. oec. Willy Oggier, Gesundheitsökonom

- Dr. med. Walter Grete, Past-Präsident Ärztegesellschaft des Kanton Zürich AGZ

- Dr. med. Urs Stoffel, Präsident der AGZ, Mitglied des Zentralvorstands der FMH

- Dr. med. Josef Widler, Vizepräsident der AGZ, Moderator des Abends

- Die teilnehmenden Ärztinnen und Ärzte

Aktuelle Forumthemen
Diskutieren Sie mit! Im Forum präsentieren wir regel-
mässig brisante Themen aus Politik, Ökonomie
und Wissenschaft, die das Schweizer Gesundheitswesen
betreffen. Bringen Sie Ihre Meinung ein oder kom-
mentieren Sie die Äusserungen Ihrer Kolleginnen und
Kollegen. Das Forum finden Sie unter:
www.saez.ch/forum/

